\title{
Association of Serum Bilirubin Levels With Sarcopenia in Patients With Type 2 Diabetes: a Cross-Sectional Study in China
}

\section{Chuan Wang}

Department of Endocrinology, Qilu hospital

Chen Jin

Department of Endocrinology, Maternity and Child Health Care of Zaozhuang

Jidong Liu

Department of Endocrinology, Qilu hospital

\section{Xiaofei Yin}

Department of Endocrinology, Qilu hospital

\section{Chuanlong Wu}

Department of Endocrinology, Qilu hospital

Jinbo Liu ( $\nabla$ jinboliu@sdu.edu.cn )

Shandong University https://orcid.org/0000-0002-5069-2450

\section{Research article}

Keywords: Bilirubin, Sarcopenia, Type 2 diabetes mellitus, Men

Posted Date: August 19th, 2020

DOl: https://doi.org/10.21203/rs.3.rs-45909/v1

License: (c) (1) This work is licensed under a Creative Commons Attribution 4.0 International License. Read Full License 


\section{Abstract}

Background: The prevalence of sarcopenia increased in type 2 diabetes mellitus (T2DM). Elevated oxidative stress and inflammation played important roles in the pathogenesis of diabetic sarcopenia. Bilirubin had been confirmed to possess potential anti-oxidative activity. This study aimed to explore the relationship between bilirubin and sarcopenia in patients with T2DM.

Methods: A total of 251 patients (124 men and 127 postmenopausal women) with T2DM aged $\geq 50$ years were recruited. Levels of serum total bilirubin (TBIL), direct bilirubin (DBIL) and indirect bilirubin (IBIL) were measured. Muscle mass was measured by dual-energy X-ray absorptiometry (DXA). The relationships between serum bilirubin levels and appendicular skeletal muscle mass index (SMI) were assessed using a Pearson correlation analysis and multiple linear regression analysis in men and women, respectively. Multiple logistic regression analysis was used to evaluate the association of the highest quartile of serum bilirubin levels with sarcopenia.

Results: TBIL $(r=0.211, p=0.019)$ and IBIL $(r=0.249, p=0.005)$ were positively associated with SMI in men, but not in women. After multiple adjustment in multiple linear regression analysis, TBIL $(p=0.023)$ and IBIL $(p=0.012)$ were also significantly associated with SMI in men. In multiple logistic regression analysis, the highest quartile of IBIL showed a decreased OR for sarcopenia $(O R=0.243, P=0.009)$ in men.

Conclusions: Both of TBIL and IBIL were positively associated with muscle mass in men with T2DM. IBIL might have a stronger protective effect on the occurrence of sarcopenia in men with T2DM.

\section{Background}

Sarcopenia is characterized by low muscle mass, low muscle strength and low physical performance [1]. The prevalence of sarcopenia ranged from $10-40 \%$ in healthy adults aged $\geq 60$ [2]. Patients with sarcopenia had increased risk for frailty, falls, fracture and even mortality [3, 4]. Therefore, screening risk factors and developing effective treatments for sarcopenia is extremely important. By 2017, it was estimated that 451 million people worldwide were suffering from diabetes and approximately $90 \%$ were type 2 diabetes mellitus (T2DM) [5]. It has been well-established that T2DM could increase the risk of sarcopenia [6-9]. However, the mechanism of sarcopenia remains unclear.

It is well known that elevated oxidative stress and inflammation play important roles in the pathogenesis of diabetic sarcopenia [10]. Bilirubin, the end product of heme metabolism, has been recently confirmed to play a protective role in metabolic syndrome and diabetes through antagonizing oxidative stress and chronic inflammation [11-14]. Till now, few studies explored the relationship between bilirubin levels and sarcopenia and no definite conclusion had been reached [15, 16]. Kawamoto et al. [15] found that increased serum total bilirubin (TBIL) was associated with increased handgrip strength in communitydwelling Japanese adults. Kim et al. [16] observed no association of TBIL with sarcopenia in patients with chronic liver diseases after multivariate adjustment. But the relationship between bilirubin levels and 
sarcopenia in patients with T2DM has not been explored. So, we conducted this cross-sectional study to explore whether bilirubin levels were in association with sarcopenia in middle-aged and elderly patients with T2DM.

\section{Methods}

\section{Study participants}

This cross-sectional study included 251 patients (124 men and 127 postmenopausal women) with T2DM aged $\geq 50$ years at Qilu Hospital of Shandong University from January 2017 to December 2019. Patients with severe liver disease (liver cirrhosis or apparently abnormal liver function defined as serum aspartate aminotransferase (AST) or alanine aminotransferase (ALT) levels were higher than $120 \mathrm{U} / \mathrm{L}$ ), severe kidney disease, any malignant disease or hematologic disease were excluded. Patients with apparently abnormal bilirubin levels (higher than two times the upper limit of the normal range) were excluded. Diabetes was diagnosed based on the 2006 World Health Organization (WHO) criteria [17]. The study protocol was in strict accordance with the provisions of the Declaration of Helsinki II and had been approved by the ethics committee of Qilu Hospital of Shandong University. All participants provided written informed consent.

\section{Data Collection}

The computerized patient medical record system of Qilu Hospital of Shandong University was used to collect basic demographic data of all patients, including age, gender, height, weight, blood pressure (BP) and smoking history. Fasting venous blood were collected for the measurement of FBG, HbA1c, total cholesterol (TC), triglyceride (TG), ALT, AST, creatinine, TBIL, direct bilirubin (DBIL) and indirect bilirubin (IBIL). HOMA2-IR was calculated using FBG and fasting C-peptide according to the computerized formula from http://www.ocdem.ox.ac.uk/ [18]. Appendicular skeletal muscle mass (ASM) and body fat percentage were measured by the dual-energy X-ray absorptiometry (DXA) scanner. The appendicular skeletal muscle mass index (SMI) was calculated as: SMI $\left(\mathrm{kg} / \mathrm{m}^{2}\right)=\mathrm{ASM}(\mathrm{kg}) / \mathrm{height}^{2}\left(\mathrm{~m}^{2}\right)$. Sarcopenia was defined as SMI lower $7.0 \mathrm{~kg} / \mathrm{m}^{2}$ (men) and $5.4 \mathrm{~kg} / \mathrm{m}^{2}$ (women) according to Asian Working Group for Sarcopenia (AWGS) [1]. The eGFR was calculated based on Chronic Kidney Disease Epidemiology Collaboration (CKD-EPI) equation [19].

\section{Statistical analysis}

Kolmogorov-Smirnov test was used to test the normal distribution of the data. The continuous variables with normal distribution and non-normal distribution were expressed as the mean \pm standard deviation (SD) and the median (interquartile range), respectively. Student's t-test (variables with normal distribution) or Mann-Whitney U-test (variables with non-normal distribution) were used to compare the differences between two groups. The relationships between serum bilirubin levels and SMI were assessed using a Pearson correlation analysis and multiple linear regression analysis in men and women, respectively. Multiple logistic regression analysis was used to determine the association of the highest quartile of 
serum bilirubin levels with sarcopenia. All statistical analyses were performed using SPSS 23.0 software (SPSS Inc., Chicago, USA) and $P<0.05$ was considered statistically significant.

\section{Results}

\section{General characteristics of participants}

A total of 124 men and 127 postmenopausal women were enrolled. As shown in Table 1, the men with sarcopenia were older and expressed significantly lower BMI, DBP, fasting C-peptide, HOMA2-IR and TG compared with those without sarcopenia. In women, patients with sarcopenia also showed higher age and lower BMI and TC, compared with patients without sarcopenia. However, the serum bilirubin levels (including TBIL, DBIL, IBIL) were not significantly different between sarcopenia and non-sarcopenia groups both in men and women. 
Table 1

General characteristics of study participants categorized by gender and sarcopenia.

\begin{tabular}{|c|c|c|c|c|c|c|}
\hline \multirow[t]{2}{*}{ Characteristics } & \multicolumn{3}{|l|}{ Men } & \multicolumn{3}{|l|}{ Women } \\
\hline & Sarcopenia & $\begin{array}{l}\text { Non- } \\
\text { sarcopenia }\end{array}$ & $\begin{array}{l}P \\
\text { value }\end{array}$ & Sarcopenia & $\begin{array}{l}\text { Non- } \\
\text { sarcopenia }\end{array}$ & $\begin{array}{l}P \\
\text { value }\end{array}$ \\
\hline $\mathrm{N}$ & 51 & 73 & - & 38 & 89 & - \\
\hline Age (years) & $\begin{array}{l}62.61 \pm \\
6.93\end{array}$ & $\begin{array}{l}59.68 \pm \\
5.97\end{array}$ & 0.013 & $\begin{array}{l}65.32 \pm \\
6.22\end{array}$ & $\begin{array}{l}61.40 \pm \\
6.47\end{array}$ & 0.002 \\
\hline BMI $\left(\mathrm{kg} / \mathrm{m}^{2}\right)$ & $\begin{array}{l}24.13 \pm \\
2.94\end{array}$ & $\begin{array}{l}28.07 \pm \\
3.23\end{array}$ & $凶 0.001$ & $\begin{array}{l}22.29 \pm \\
2.83\end{array}$ & $\begin{array}{l}25.96 \pm \\
3.65\end{array}$ & $\llbracket 0.001$ \\
\hline $\mathrm{SBP}(\mathrm{mmHg})$ & $\begin{array}{l}131.86 \pm \\
20.01\end{array}$ & $\begin{array}{l}137.22 \pm \\
19.68\end{array}$ & 0.141 & $\begin{array}{l}136.87 \pm \\
23.30\end{array}$ & $\begin{array}{l}140.16 \pm \\
17.10\end{array}$ & 0.377 \\
\hline $\mathrm{DBP}(\mathrm{mmHg})$ & $\begin{array}{l}76.10 \pm \\
10.94\end{array}$ & $\begin{array}{l}81.11 \pm \\
11.02\end{array}$ & 0.014 & $\begin{array}{l}75.03 \pm \\
12.41\end{array}$ & $\begin{array}{l}77.91 \pm \\
10.88\end{array}$ & 0.192 \\
\hline FBG (mmol/L) & $7.69 \pm 2.91$ & $8.20 \pm 2.62$ & 0.317 & $8.53 \pm 2.84$ & $8.11 \pm 2.89$ & 0.463 \\
\hline $\mathrm{HbA}_{1 \mathrm{c}}(\%)$ & $8.17 \pm 1.60$ & $8.36 \pm 1.92$ & 0.562 & $8.87 \pm 2.17$ & $8.69 \pm 1.86$ & 0.632 \\
\hline $\begin{array}{l}\text { Fasting C-peptide } \\
(\mathrm{ng} / \mathrm{mL})\end{array}$ & $\begin{array}{l}0.93(0.71 \\
\text { to } 1.72)\end{array}$ & $\begin{array}{l}1.55(1.00 \\
\text { to } 2.09)\end{array}$ & 0.010 & $\begin{array}{l}1.23(0.64 \\
\text { to } 1.63)\end{array}$ & $\begin{array}{l}1.13(0.71 \\
\text { to } 1.71)\end{array}$ & 0.836 \\
\hline HOMA2-IR (unit) & $\begin{array}{l}0.80(0.56 \\
\text { to } 1.41)\end{array}$ & $\begin{array}{l}1.29(0.79 \\
\text { to } 1.92)\end{array}$ & 0.013 & $\begin{array}{l}1.02(0.55 \\
\text { to } 1.50)\end{array}$ & $\begin{array}{l}0.99(0.57 \\
\text { to } 1.46)\end{array}$ & 0.945 \\
\hline TG (mmol/L) & $\begin{array}{l}1.32(0.98 \\
\text { to } 1.86)\end{array}$ & $\begin{array}{l}1.58(1.19 \\
\text { to } 2.28)\end{array}$ & 0.026 & $\begin{array}{l}1.46(0.97 \\
\text { to } 2.12)\end{array}$ & $\begin{array}{l}1.40(1.06 \\
\text { to } 1.95)\end{array}$ & 0.941 \\
\hline $\mathrm{TC}(\mathrm{mmol} / \mathrm{L})$ & $4.13 \pm 0.95$ & $4.49 \pm 1.01$ & 0.050 & $5.09 \pm 1.06$ & $4.68 \pm 1.01$ & 0.044 \\
\hline $\begin{array}{l}\text { Creatinine } \\
(\mu \mathrm{mol} / \mathrm{L})\end{array}$ & $\begin{array}{l}72.64 \pm \\
17.54\end{array}$ & $\begin{array}{l}74.51 \pm \\
19.63\end{array}$ & 0.590 & $\begin{array}{l}55.37 \pm \\
15.66\end{array}$ & $\begin{array}{l}56.03 \pm \\
12.88\end{array}$ & 0.803 \\
\hline $\begin{array}{l}\text { eGFR } \\
\left(\mathrm{mL} / \mathrm{min} / 1.73 \mathrm{~m}^{2}\right)\end{array}$ & $\begin{array}{l}93.84 \pm \\
15.02\end{array}$ & $\begin{array}{l}94.28 \pm \\
15.55\end{array}$ & 0.876 & $\begin{array}{l}92.53 \pm \\
14.24\end{array}$ & $\begin{array}{l}94.31 \pm \\
12.46\end{array}$ & 0.482 \\
\hline $\mathrm{SMI}\left(\mathrm{kg} / \mathrm{m}^{2}\right)$ & $6.33 \pm 0.48$ & $7.72 \pm 0.58$ & $凶 0.001$ & $4.88 \pm 0.30$ & $6.14 \pm 0.65$ & $\nabla 0.001$ \\
\hline $\begin{array}{l}\text { Body fat } \\
\text { percentage (\%) }\end{array}$ & $\begin{array}{l}27.97 \pm \\
4.61\end{array}$ & $\begin{array}{l}29.14 \pm \\
4.42\end{array}$ & 0.158 & $\begin{array}{l}35.32 \pm \\
5.11\end{array}$ & $\begin{array}{l}36.04 \pm \\
4.60\end{array}$ & 0.433 \\
\hline Smoking (n, \%) & 20 (39.2\%) & 40 (54.8\%) & 0.102 & $0(0.0 \%)$ & $0(0.0 \%)$ & 1.000 \\
\hline
\end{tabular}

Abbreviations: BMI, body mass index; SBP, systolic blood pressure; DBP, diastolic blood pressure; FBG, fasting blood glucose; HOMA2-IR, homeostasis model assessment of insulin resistance; TG, triglyceride; TC, total cholesterol; eGFR, estimated glomerular filtration rate; SMI, appendicular skeletal muscle mass index; AST, aspartate aminotransferase; ALT, alanine aminotransferase; TBIL, total bilirubin; DBIL, direct bilirubin; IBIL, indirect bilirubin. Significant $P$ values $(<0.05)$ are indicated in bold. 


\begin{tabular}{|c|c|c|c|c|c|c|}
\hline \multirow[t]{2}{*}{ Characteristics } & \multicolumn{3}{|l|}{ Men } & \multicolumn{3}{|l|}{ Women } \\
\hline & Sarcopenia & $\begin{array}{l}\text { Non- } \\
\text { sarcopenia }\end{array}$ & $\begin{array}{l}P \\
\text { value }\end{array}$ & Sarcopenia & $\begin{array}{l}\text { Non- } \\
\text { sarcopenia }\end{array}$ & $\begin{array}{l}P \\
\text { value }\end{array}$ \\
\hline AST (U/L) & $\begin{array}{l}19.06 \pm \\
8.97\end{array}$ & $\begin{array}{l}20.48 \pm \\
8.39\end{array}$ & 0.369 & $\begin{array}{l}20.84 \pm \\
13.53\end{array}$ & $\begin{array}{l}19.73 \pm \\
7.17\end{array}$ & 0.634 \\
\hline $\operatorname{ALT}(\mathrm{U} / \mathrm{L})$ & $\begin{array}{l}19.12 \pm \\
13.20\end{array}$ & $\begin{array}{l}22.51 \pm \\
13.09\end{array}$ & 0.160 & $\begin{array}{l}16.74 \pm \\
12.33\end{array}$ & $\begin{array}{l}19.72 \pm \\
12.13\end{array}$ & 0.209 \\
\hline TBIL (umol/L) & $\begin{array}{l}11.19 \pm \\
4.94\end{array}$ & $\begin{array}{l}12.32 \pm \\
4.69\end{array}$ & 0.199 & $\begin{array}{l}10.25 \pm \\
4.64\end{array}$ & $9.73 \pm 4.13$ & 0.530 \\
\hline DBIL (umol/L) & $3.86 \pm 1.60$ & $3.94 \pm 1.45$ & 0.763 & $3.56 \pm 2.05$ & $3.23 \pm 1.15$ & 0.265 \\
\hline IBIL (umol/L) & $7.33 \pm 3.48$ & $8.37 \pm 3.42$ & 0.099 & $6.70 \pm 3.14$ & $6.49 \pm 3.07$ & 0.729 \\
\hline \multicolumn{7}{|c|}{$\begin{array}{l}\text { Abbreviations: BMI, body mass index; SBP, systolic blood pressure; DBP, diastolic blood pressure; FBG } \\
\text { fasting blood glucose; HOMA2-IR, homeostasis model assessment of insulin resistance; TG, } \\
\text { triglyceride; TC, total cholesterol; eGFR, estimated glomerular filtration rate; SMI, appendicular skeletal } \\
\text { muscle mass index; AST, aspartate aminotransferase; ALT, alanine aminotransferase; TBIL, total } \\
\text { bilirubin: DBIL, direct bilirubin: IBIL. indirect bilirubin Sianificant } P \text { values }(<0.05) \text { are indicated in bold }\end{array}$} \\
\hline
\end{tabular}

\section{Correlation between serum bilirubin levels and SMI in men and women}

In order to explore the relationship between serum bilirubin levels and SMI, a Pearson correlation analysis was conducted in men and women, respectively. As shown in Fig. 1, TBIL $(r=0.211, p=0.019)$ and IBIL ( $r$ $=0.249, p=0.005$ ) were positively associated with SMI in men. However, no significantly correlations between serum bilirubin levels and SMI were observed in women. Then, we conducted multiple linear regression analysis to further explore the correlation between serum bilirubin levels and SMI. As shown in Table 2, only a close association of TBIL and IBIL with SMI was observed in men. Even after adjusting for age, SBP, HbA1c, HOMA2-IR, TG, TC, eGFR, smoking and body fat percentage, TBIL $(p=0.023)$ and IBIL ( $p$ $=0.012$ ) also significantly associated with SMI in men. No significant correlations between serum bilirubin levels and SMI were observed in women. 
Table 2

Multiple linear regression analysis of serum bilirubin levels and SMI in men and women.

\begin{tabular}{|lllll|}
\hline & Model 1 & & Model 2 \\
\hline Independent variable & $\beta$ Coefficient $(95 \% \mathrm{Cl})$ & $P$ & $\beta$ Coefficient $(95 \% \mathrm{Cl})$ & $P$ \\
\hline Men & & & & \\
\hline TBIL & $0.038(0.006$ to 0.070$)$ & 0.019 & $0.037(0.005$ to 0.070$)$ & 0.023 \\
\hline DBIL & $0.057(-0.046$ to 0.160$)$ & 0.275 & $0.079(-0.029$ to 0.187$)$ & 0.152 \\
\hline IBIL & $0.063(0.019$ to 0.107$)$ & 0.005 & $0.057(0.013$ to 0.101$)$ & 0.012 \\
\hline Women & & & & \\
\hline TBIL & $7.846 E-5(-0.034$ to 0.034$)$ & 0.996 & $0.006(-0.027$ to 0.039$)$ & 0.729 \\
\hline DBIL & $-0.018(-0.115$ to 0.079$)$ & 0.714 & $2.936 E-5(-0.095$ to 0.096$)$ & 1.000 \\
\hline IBIL & $0.004(-0.043$ to 0.051) & 0.860 & $0.011(-0.035$ to 0.058$)$ & 0.631 \\
\hline Model 1: unadjusted. & & & & \\
\hline Model 2: adjusted for age, SBP, HbA1c, HOMA2-IR, TG, TC, eGFR, smoking and body fat percentage. \\
\hline
\end{tabular}

\section{Multiple logistic regression analysis of serum bilirubin levels and sarcopenia in men and women}

Finally, we conducted multiple logistic regression analysis to explore the association of TBIL and IBIL with sarcopenia since only TBIL and IBIL were significantly associated with SMI. The participants were divided by quartiles of TBIL and IBIL in men and women, respectively. The highest quartile of TBIL and IBIL were included in analysis as independent variables. As shown in Table 3, before adjustment, only the highest quartile of IBIL showed a decreased OR for sarcopenia (OR $=0.380, p=0.035)$ in men. After adjusting for age, SBP, HbA1c, HOMA2-IR, TG, TC, eGFR, smoking and body fat percentage, the decreased OR was also observed $(\mathrm{OR}=0.243, p=0.009)$. However, TBIL did not present a significant association with sarcopenia any more in men. Both of TBIL and IBIL were not associated with sarcopenia in women. 
Table 3

Multiple logistic regression analysis of serum bilirubin levels and sarcopenia in men and women.

Model 1

Odds ratio $(95 \% \mathrm{Cl})$
Model 2

Independent variable

Men

The highest quartile of TBIL

$0.646(0.273$ to 1.530$)$

0.321

$0.424(0.153$ to 1.175$)$

0.099

The highest quartile of IBIL

0.380 (0.155 to 0.933$)$

0.035

0.243 (0.084 to 0.703 )

0.009

Women

The highest quartile of TBIL

0.841 (0.348 to 2.031 )

0.700

0.825 (0.297 to 2.290$)$

0.711

The highest quartile of IBIL

0.891 (0.367 to 2.159)

0.798

0.829 (0.305 to 2.254$)$

0.714

Model 1: unadjusted.

Model 2: adjusted for age, SBP, HbA1c, HOMA2-IR, TG, TC, eGFR, smoking and body fat percentage.

\section{Discussion}

With aging, there is a rise in the prevalence of metabolic and musculoskeletal diseases, including T2DM and sarcopenia $[5,20]$. The bidirectional relationship between T2DM and sarcopenia has been demonstrated in a previous review [10]. T2DM is characterized by insulin resistance, an inflammatory phenotype, oxidative stress and increased advanced glycation end-products (AGEs), which may lead to cell death and further losses in skeletal muscle mass, strength, and function, finally facilitating the occurrence of sarcopenia [10]. Bilirubin is one of the most active endogenous antioxidant molecules and has been proved beneficial to the prevention and treatment of T2DM [11-14, 21]. However, the role of bilirubin in the pathophysiology of sarcopenia in patients with T2DM has not been explored. In order to clarify the relationship between serum bilirubin levels and sarcopenia, we conducted this cross-sectional study and performed it in different gender.

Unexpectedly, the present study showed that the levels of TBIL, DBIL and IBIL were not significantly different between sarcopenia group and non-sarcopenia group. The serum bilirubin levels were lowered by smoking and negatively associated with BMI [22-24]. As shown in Table 1, sarcopenia group presented a lower BMI and relatively lower proportion of smoker than that in non-sarcopenia group in men. Theoretically speaking, the serum bilirubin levels of sarcopenia group should be apparently higher than that of non-sarcopenia group. However, the results were just on the contrary. This paradoxical phenomenon instead suggested the possible correlation of serum bilirubin levels with sarcopenia. Then, a correlation analysis was performed. As shown in Fig. 1, we found that TBIL and IBIL positively associated with SMI in men, but not in women, which was further supported by further multiple linear regression analysis. However, in multiple logistic regression analysis, only the highest quartile of IBIL showed a decreased OR for sarcopenia after multiple adjustment in men. These data indicated that IBIL, but not DBIL, played an important role in protecting against the occurrence of sarcopenia in men with T2DM. 
TBIL is the sum of DBIL and IBIL, and most serum bilirubin is IBIL [25]. Therefore, we supposed that serum IBIL played a major role in the protection of sarcopenia, although both IBIL and DBIL had the properties of antioxidant [26]. Besides, the different clinic significance between DBIL and IBIL can also be observed in other diseases. Gilbert syndrome patients with mildly elevated IBIL had lower risk of coronary vascular diseases, while this association was not observed in Dubin-Johnson syndrome with elevated DBIL [27].

The close relationship between IBIL and sarcopenia was only observed in men. The gender difference might be attributed to the different pathophysiology of serum bilirubin and sarcopenia between men and women. Several studies had found that men had higher serum bilirubin levels than women [28]. Besides, serum bilirubin levels could also be affected by estrogens [29]. Additionally, a sex-associated pattern of aging might have different effects on muscle mass and quality, including sex hormones [30]. An agreement had been reached that sex hormones were involved in maintaining skeletal homoeostasis: both of testosterone and estrogen could promote muscle protein synthesis, and low levels of testosterone and estrogen were associated with sarcopenia [31, 32]. Moreover, in women, age-induced changes in muscle were mostly expressed in muscle quality; while men mostly expressed in muscle mass decline [32]. All the above differences between genders might affect the relationship between bilirubin and sarcopenia in men and women.

Besides, the present study also has some limitations. First, because of the cross-sectional nature of this study, causality between serum bilirubin levels and sarcopenia onset could not be confirmed. Second, the participants of this study were Chinese with age $\geq 50$ years and the samples were relatively limited. Third, the sarcopenia was defined only based on SMI. Muscle strength and physical performance were not assessed. Finally, some other potential influence factors of sarcopenia, such as sex hormones, inflammatory biomarkers, exercise, were not fully adjusted, which might affect the accuracy of the results.

\section{Conclusions}

Collectively, data from this study indicated that TBIL and IBIL were positively associated with SMI in men with T2DM, and IBIL might be more protective of sarcopenia development and progression. More detailed studies are necessary to further define the pathophysiological roles of bilirubin in sarcopenia.

\section{Abbreviations}

T2DM: type 2 diabetes mellitus; TBIL: total bilirubin; AST: aspartate aminotransferase; ALT: alanine aminotransferase; WHO: World Health Organization; BP: blood pressure; TC: total cholesterol; TG: triglyceride; DBIL: direct bilirubin; IBIL: indirect bilirubin; ASM: Appendicular skeletal muscle mass; DXA: dual-energy X-ray absorptiometry; SMI: skeletal muscle mass index; AWGS: Asian Working Group for Sarcopenia; CKD-EPI: Chronic Kidney Disease Epidemiology Collaboration; AGEs: advanced glycation end-products. 


\section{Declarations}

Acknowledgements

The author thanks all team members and participants in this study.

\section{Authors' contributions}

JBL and CW designed the study and drafted the initial manuscript. JDL and CJ collected the data. CW, $\mathrm{XFY}$, and CLW conducted analysis. All authors approved the final manuscript.

\section{Funding}

The present study was supported by National Natural Science Foundation of China (No.81700739).

\section{Availability of data and material}

The data used to support the findings of this study are available from the corresponding author upon request.

\section{Ethics approval and consent to participate}

The study protocol was in strict accordance with the provisions of the Declaration of Helsinki II and had been approved by the ethics committee of Qilu Hospital of Shandong University. All participants provided written informed consent.

\section{Consent of publication}

No applicable.

\section{Competing interests}

The authors declare that they have no competing interests.

\section{References}

1. Chen LK, Liu LK, Woo J, et al. Sarcopenia in Asia: consensus report of the Asian Working Group for Sarcopenia. J Am Med Dir Assoc. 2014; 15: 95-101.

2. Amog K, Raina P, Mayhew AJ, et al. The prevalence of sarcopenia in community-dwelling older adults, an exploration of differences between studies and within definitions: a systematic review and meta-analyses. Age Ageing. 2018; 48: 48-56.

3. Abellan van Kan G. Epidemiology and consequences of sarcopenia. J Nutr Health Aging. 2009; 13: 708-712. 
4. Rolland Y, Czerwinski S, Abellan Van Kan G, et al. Sarcopenia: its assessment, etiology, pathogenesis, consequences and future perspectives. J Nutr Health Aging. 2008; 12: 433-450.

5. Cho NH, Shaw JE, Karuranga S, et al. IDF diabetes atlas: global estimates of diabetes prevalence for 2017 and projections for 2045. Diabetes Res Clin Pract. 2018; 138: 271-281.

6. Wang T, Feng $X$, Zhou J, et al. Type 2 diabetes mellitus is associated with increased risks of sarcopenia and pre-sarcopenia in Chinese elderly. Sci Rep. 2016; 6: 38937.

7. Kim TN, Park MS, Yang SJ, et al. Prevalence and determinant factors of sarcopenia in patients with type 2 diabetes. Diabetes Care. 2010; 33: 1497.

8. Park SW, Goodpaster BH, Lee JS, et al. Excessive loss of skeletal muscle mass in older adults with type 2 diabetes. Diabetes Care. 2009; 32: 1993-1997.

9. Yang L, Smith L, Hamer M. Gender-specific risk factors for incident sarcopenia: 8-year follow-up of the English longitudinal study of ageing. J Epidemiol Community Health. 2019; 73: 86.

10. Mesinovic J, Zengin A, De Courten B, Ebeling PR, Scott D. Sarcopenia and type 2 diabetes mellitus: a bidirectional relationship. Diabetes Metab Syndr Obes. 2019; 12: 1057-1072.

11. Inoguchi T, Sonoda N, Maeda Y. Bilirubin as an important physiological modulator of oxidative stress and chronic inflammation in metabolic syndrome and diabetes: a new aspect on old molecule. Diabetol Int. 2016; 7: 338-341.

12. Nano J, Muka T, Cepeda M, et al. Association of circulating total bilirubin with the metabolic syndrome and type 2 diabetes: A systematic review and meta-analysis of observational evidence. Diabetes Metab. 2016; 42: 389-397.

13. Zhong P, Sun D, Wu D, Liu X. Total bilirubin is negatively related to diabetes mellitus in Chinese elderly: a community study. Ann Transl Med. 2019; 7: 474.

14. Wu Y, Li M, Xu M, et al. Low serum total bilirubin concentrations are associated with increased prevalence of metabolic syndrome in Chinese. J Diabetes. 2011; 3: 217-224.

15. Kawamoto R, Ninomiya D, Kumagi T. Handgrip Strength Is Positively Associated with Mildly Elevated Serum Bilirubin Levels among Community-Dwelling Adults. Tohoku J Exp Med. 2016; 240: 221-226.

16. Hyun Kim K, Kyung Kim B, Yong Park J, et al. Sarcopenia assessed using bioimpedance analysis is associated independently with significant liver fibrosis in patients with chronic liver diseases. Eur $\mathrm{J}$ Gastroenterol Hepatol. 2020; 32: 58-65.

17. WHO. Definition and diagnosis of diabetes mellitus and intermediate hyperglycaemia: A report of WHO/ IDF consultation. Geneva WHO 2006.

18. Levy JC, Matthews DR, Hermans MP. Correct homeostasis model assessment (HOMA) evaluation uses the computer program. Diabetes Care. 1998; 21: 2191-2192.

19. Levey AS, Stevens LA, Schmid CH, et al. A new equation to estimate glomerular filtration rate. Ann Intern Med. 2009; 150: 604-612.

20. Briggs AM, Woolf AD, Dreinhöfer K, et al. Reducing the global burden of musculoskeletal conditions. Bull World Health Organ. 2018; 96: 366-368. 
21. Gazzin S, Vitek L, Watchko J, Shapiro SM, Tiribelli C. A Novel Perspective on the Biology of Bilirubin in Health and Disease. Trends Mol Med. 2016; 22: 758-768.

22. Breimer LH, Mikhailidis DP. Does bilirubin protect against developing diabetes mellitus?. J Diabetes Complications. 2016; 30: 728-737.

23. Kodal JB, Çolak Y, Kobylecki CJ, et al. Smoking Reduces Plasma Bilirubin: Observational and Genetic Analyses in the Copenhagen General Population Study. Nicotine Tob Res. 2020; 22: 104-110.

24. Takei R, Inoue T, Sonoda N, et al. Bilirubin reduces visceral obesity and insulin resistance by suppression of inflammatory cytokines. PLoS One. 2019; 14: e0223302.

25. Fevery J. Bilirubin in clinical practice: a review. Liver Int. 2008; 28: 592-605.

26. Wu TW, Fung KP, Wu J, Yang CC, Weisel RD. Antioxidation of human low density lipoprotein by unconjugated and conjugated bilirubins. Biochem Pharmacol. 1996; 51: 859-862.

27. Bulmer AC, Verkade HJ, Wagner KH. Bilirubin and beyond: a review of lipid status in Gilbert's syndrome and its relevance to cardiovascular disease protection. Prog Lipid Res. 2013; 52: 193-205.

28. Lee SG, Lee W, Kim JH, Kwon OH. Gender-specific reference intervals for serum total bilirubin in healthy Korean adults. Clin Biochem. 2012; 45: 1257-1259.

29. Walden CE, Knopp RH, Johnson JL, et al. Effect of estrogen/progestin potency on clinical chemistry measures. The lipid research clinics program prevalence study. Am J Epidemiol. 1986; 123: 517-531.

30. Di Monaco M, Castiglioni C, Vallero F, Di Monaco R, Tappero R. Sarcopenia is more prevalent in men than in women after hip fracture: a cross-sectional study of 591 inpatients. Arch Gerontol Geriatr. 2012; 55: e48-e52.

31. Seo DH, Lee YH, Park SW, et al. Sarcopenia is associated with non-alcoholic fatty liver disease in men with type 2 diabetes. Diabetes Metab. 2019; S1262-3636(19)30160-0.

32. Messier V, Rabasa-Lhoret R, Barbat-Artigas $\mathrm{S}$, et al. Menopause and sarcopenia: A potential role for sex hormones. Maturitas. 2011; 68: 331-336.

\section{Figures}




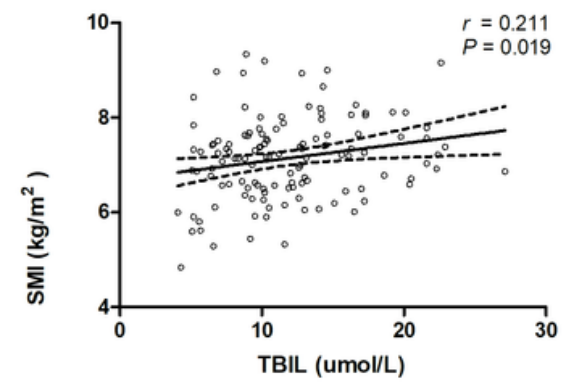

A

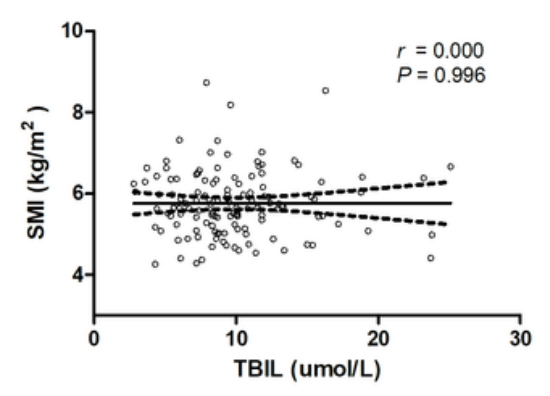

D

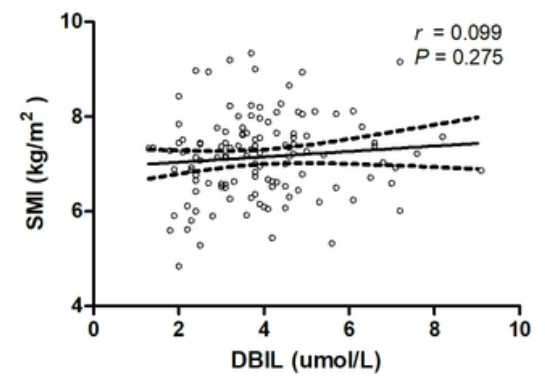

B

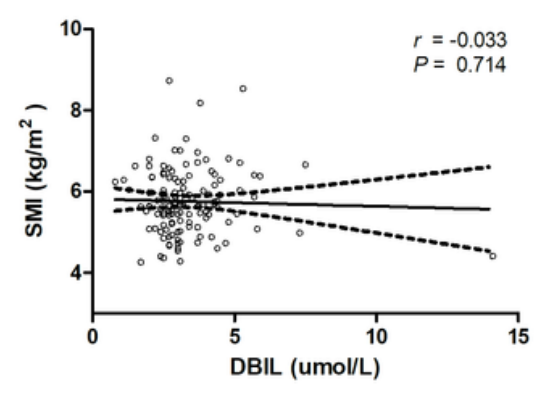

E

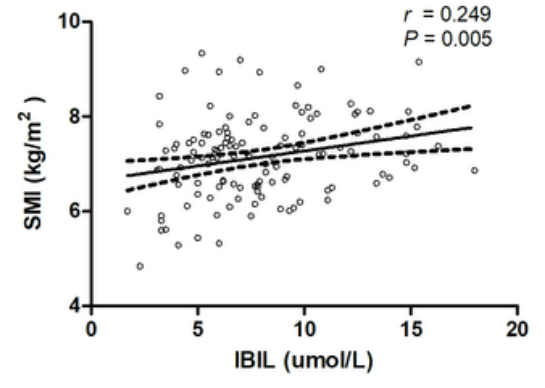

C

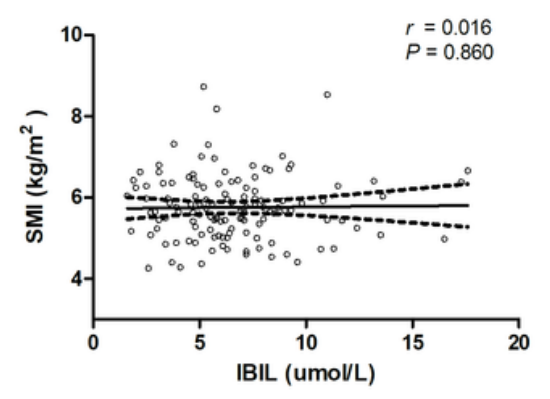

$\mathbf{F}$

Figure 1

Correlation between serum bilirubin levels and $S M I$ in men $(A, B, C)$ and women $(D, E, F)$. SMI = appendicular skeletal muscle mass index. 